\title{
Connectivity...... Teleradiology and beyond (Part one)
}

C onnectivity, Teleradiology and Telemedicine. These are some of the "buzz words" describing the latest technology in our field and, as with any new technology, it has spawned its own jargon and set of acronyms. This article aims to explain some of the jargon and in so doing enhance the level of discussion of this important topic.

\section{Why
connectivity?}

Throughout the world health care providers are finding that improved productivity and efficiency are critical to long term success. The use of connectivity has the following primary goals:

- Connect existing digital equipment in order to reduce manual activities and increase departmental efficiency.

- Moveimagesquickly throughout thefacility to enhance patient care.

- Moveimage data between facilities in order to make use of centres of excellence to speed diagnosis.

- Integrate image data with diagnostic information to improve the diagnostic process.

- To allow disadvantaged communities access, via telecommunications, to specialistcare.

One of the most common terms that is being bandied about is DICOM. What is DICOM and why is it so important?

DICOM is an acronym for Digital
Imaging and Communications in Medicine.DICOMwas developed by theAmerican College of Radiology (ACR) in conjunction with the National Electrical Manufacturers' Association (NEMA) in America. The current version of the standard is "version 3,0".

We are talking here of connecting two devices together, and as far as DICOM is concerned that means we have to describe both ends of the connection. This leads to the concept of Service Classes. Service Classes provide very specific information about the kinds of images to be exchanged and the expected operation of the devices on both ends of the connection. An alternative description of a Service Classis aSOP Class (SOPstandsfor Service/ObjectPair). Images are called "objects" and the operations the device is to perform on the images are called "services". In addition, devices claim conformance to a Service Class as either a User or a Provider.

By way of another example, laser printers areProviders of the Basic GreyscalePrint ManagementService Class.
DICOM requires the manufacturer to publish a Conformance Statement for all devices that claim conformance to the standard. The Conformance Statement contains very specific information about the device. To use the laser printer example above, the Conformance Statement would contain the following information: - The network on which it operates, for exampleTCP/IP overEthernet

- The Service Class (or Classes) that the device conforms to, for example Basic Greyscale Print Management(This means it prints black and white images)

- The range of values supported for various functions, for example formats of l-up to 35 -up and that it can print $4096 \times 5120$ pixels

- Optional functions that are supported, such as slides

It will be clear from the above that the simple statement "DICOM compatible" doesn't mean very much and that an indepth investigation is needed to establish whether devices will indeed "talk" to each other and perform useful work.

\section{Next we look at MODEMS and ISDN}

MODEM stands for Modulator Demodulator. The most common application of a modem is the fax machine where a document is scanned and the information (characters and graphics) is modulated

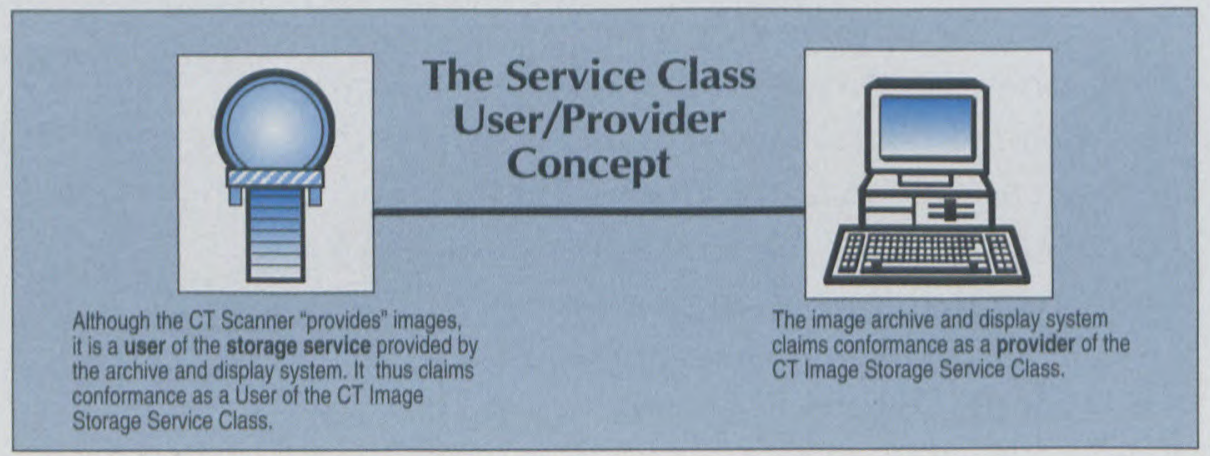

to page 43 


\section{from page 42}

onto a tone that can be transmitted down a conventional telephoneline.At thereceiving end the tone is demodulated back into characters and graphics and printed out. Standard faxes operate at 9600 bits per second (or 9600 Baud). Modems on computers that share data or access the Internetcan run at up to 28800 Baud, however this is approaching the limit of what a standard telephone line can handle.

The next generation of telephonelines will use Integrated Services Digital Network (ISDN). When you pick up your phone, the dial tone you hear is sent from your telephone exchange which, in modem areas, is a powerful digital switch. The switch, in turn, is linked to hundreds of thousands of high speed digital connections that are capable of carrying thousands of voice and data conversations simultaneously. The problem comes in with the line that connects your phone to this network -it is analog?

As can be appreciated, this may work well for voice and faxes but when we want to transfer massive amounts of data (such as contained in a radiographicimage) we are faced with a very slow system.

ISDN is a fully digital service which allows simultaneous high-speed transfer of live video, data and fax over a conventional telephone line. ISDN comprises two types of communications channels:

\section{BChannels (Bearer Channels)}

$B$ Channels are designed to carry virtually any kind of digital information. They are normal telephone lines that are capable of carrying data at 64000 bits per second.

\section{DChannels (Delta Channels)}

DChannels carry call signalling and set-up information such as the telephone number of the network. This channel dramatically speeds up connection time.

\section{Basic Rate}

Basic rate ISDN provides the user with two $64 \mathrm{Kbps} \mathrm{B}$ channels and one $16 \mathrm{Kbps} \mathrm{D}$ channel. Basic access represents ISDN in its simplestform.

The authorwishes to thank the S.A.ISDN Forum, GEMedical, Tecmed (Kodak Health Systems), S.A.Philips and Siemens for providinginformation for this article.

\section{COMPANYNEWS}

PHILIPS MEDICAL

\section{Telemedicine - the diagnostic information highway of the future}

PhilipsMedical Systems offer a range of products, continuing development and support expertise Optimising resourcemanagement by providing professional expertise remotely, where previously no service was offered, or to achievecompetitive business advantagefor the private practitioner, will be increasingly addressed by the use of computer driven communication networks.

From the 'total concept' approach of a largeinvestment, singlePictureArchiving and Communication Systems (PACS) of the 1970s, a phased, modular approach istoday's formula for implementation. Philips Medical Systems has fully adopted a customer focused, bottom up design approach, allowing customisation to achieve specific requirements. The principle is that transmission, display, manipulation and storage of digital images should offer physicians clinical advantages, improve operation of health care institutions, and ultimately optimise patientservice.

The modular concept facilitates staged integration of the Radiology Information System (RIS), the digital information of $\mathrm{MR}, \mathrm{CT}$, angiographic and R/F applications, cassette based computed radiography and remote tele-images. The evolvement of the DICOM standard for transferring images and associated information between digital devices regardless of manufacturer, provides further expansion of the integrated modular approach.
The Philips "Tele-Imager" teleradiology system offers custom designed software. The basic system, which can be fully extended in a modular approach, comprises a capture station, a viewing station and the transmission medium. Multiple resolution choices from $1 \mathrm{~K}^{2}$ to $4 \mathrm{~K}^{2}$ film digitisation with 12 bit grey scale imaging, choice of compression factors and combined with $1600 \times 1200$ monitor display, provide superior diagnostic capability. Image transmission can take place by standard telephone lines, Diginet or ISDNlines, local or wide area networks. The ability to interconnect the system with other modalities - CT, MR, angio, R/F and computed radiography provide for an integrated network development.

For more information, contact your local Philips Medical representative, or tel.(011)4803035; e-mail mbrandt@ iafrica.com. 